Wouter H. van Geffen ${ }^{1}$, Torsten G. Blum² , Stefano Aliberti ${ }^{3,4}$, Kevin G. Blyth ${ }^{5}$, Clementine Bostantzoglou 6 , Amy Farr ${ }^{7}$, Bogdan Grigoriu8, Georgia Hardavella 9 , Rudolf M. Huber®10, Nick Maskell11, Gilbert Massard ${ }^{12}$, Najib M. Rahman ${ }^{13}$, Daiana Stolz ${ }^{14}$ and Jan van Meerbeeck ${ }^{15}$

torsten-gerriet.blum@helios-gesundheit.de

@EuroRespSoc

\title{
Editorial
}

\section{Continuous professional development: elevating thoracic oncology education in Europe}

Thoracic oncology encompasses a broad spectrum of primary tumour entities originating from various pulmonary, tracheobronchial, pleural, mediastinal and chest wall tissues or distinct cell types within these compartments. Lung cancer represents by far the most frequent malignant tumour within the thoracic cavity, and is the most common type and largest killer among all cancers worldwide. Lung cancer leads mortality statistics in Europe, accounting for at least one fifth of all cancerrelated deaths [1]. In addition, lung cancer causes a significant burden of symptoms in a population of patients with high comorbidity, providing significant challenges to national healthcare systems in the European Union, with the highest overall costs among all cancer types [2-4]. Contrary to the general stigma applied to lung cancer patients, where they are often assumed to suffer from a self-inflicted disease, it is now estimated that up to $10-20 \%$ of lung cancer patients are neversmokers $[5,6]$. Even if numerically far lower, the remaining $<5 \%$ of primary thoracic malignancies other than lung cancer challenge thoracic oncology specialists, as well general pulmonologists, often in daily practice, be they pleural mesotheliomas, thymic or neuroendocrine tumours, sarcomas or rare entities such as germ-cell tumours [7, 8].

Epoch-making progress has been made recently in thoracic oncology after decades of only minor improvements regarding prognosis. Nowadays, detailed molecular tumour profiling has led to personalised diagnostic-therapeutic sequences.
In addition, immunotherapy is developing as the most exciting and fundamental turning point in the history of lung cancer treatment. In 2009, when this step-change in highly effective therapeutic approaches was not yet foreseeable, the European Respiratory Society (ERS) implemented the farsighted decision to create its own Thoracic Oncology Assembly. The first step of a detailed, forwardlooking action plan was launched in 2010 [9]. In subsequent years, the role of the pulmonologist as pilot and partner in thoracic oncology has been introduced by the American Thoracic Society and the ERS [10].

Against this background, the Thoracic Oncology Assembly has been able to create a basis for standard educational formats and activities in line with the overall ERS strategy over the last few years. Amongst other initiatives, particularly the HERMES (Harmonised Education in Respiratory Medicine for European Specialists) programme for Thoracic Oncology, this has provided a comprehensive template in the field and is recognised worldwide as a role model [11-13]. Given the currently hyperdynamic environment, the Thoracic Oncology Assembly now faces the fascinating opportunity to promote an adapted action plan. While scientific, diagnostic and therapeutic advances are evolving rapidly, the European routine care landscape in thoracic oncology is characterised by a vast heterogeneity in available resources as well as in responsible professionals. The field of systemic therapies for patients with thoracic malignancies is one clear example: in
Cite as: van Geffen WH, Blum TG, Aliberti S, et al. Continuous professional development: elevating thoracic oncology education in Europe. Breathe 2019; 15: 279-285.

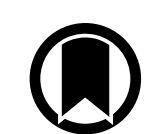

CrossMark

@ERSpublications

The @EuroRespSoc launches a new thoracic oncology continuous professional development

programme http://bit.ly/31ShuTp 
about half of European countries, the involvement of pulmonologists in systemic therapy is essential, contrasting with a significant number of nations in which pulmonologists are neither specifically trained nor active in this area [14]. Scientific progress does not pay any attention to currently established core pneumological skills in thoracic oncology. Rather, it may be envisaged that endoscopic methods will become insignificant in the medium term through the ongoing evolution of sometimes co-equal noninvasive diagnostic measures, namely the increasing maturity of liquid biopsies [15]. The advent of high-quality data demonstrating mortality reduction with lung cancer screening poses a separate but enormous challenge in quality control, implementation and rational delivery of a programme, which may transform our approach to lung cancer. Thus, there is to date an inadequately addressed need for continuous professional development (CPD) among pulmonologists to keep pace with the revolutionary trends in thoracic oncology.

\section{An innovative campaign towards a joint and vivid educational platform in thoracic oncology}

The answer to these demanding challenges is firstly one of communication. Analogous to the ERS master plan for other respiratory disease areas, our present educational thoracic oncology platform will be transformed stepwise from a fixed masterpiece to a dynamically adapting up-to-date platform. This new and unique platform is meant to arouse true thirst for knowledge in both general and specialised clinicians, and furthermore, to enable realistic, targeted $C P D$ tailored to personal needs and individual professional career stages.

This document highlights the essential steps from the development towards the aspired implementation of the new ERS educational programme in thoracic oncology, and provides insights into the underlying CPD syllabus, planned educational contents and formats as well as specific communication strategies.

From our mission to strengthen and improve the diagnosis and care of thoracic malignancies in Europe, we envisage an adaptive and evidencebased CPD matrix based on multidisciplinary education. Our educational campaign seeks to cover the entire continuum of thoracic malignancy. We aim to provide attractive educational formats to all physicians and allied health professionals with an interest in thoracic oncology, independent of original discipline, grade of specialisation or current career status. Multidimensional collaboration within and outside the ERS is key to achieve these ambitious goals, namely by:

- Horizontal linkage with other related ERS assemblies
- Ensuring multiprofessionality and patient representation by incorporating allied respiratory professionals and the European Lung Foundation, respectively

- Cooperation with other dedicated international partner societies in the field of thoracic oncology (i.e. the European Society of Radiology, the European Society of Thoracic Surgeons and the International Association for the Study of Lung Cancer)

- Vertical liaison with respiratory societies and thoracic oncology services on the national and local levels, aiming to set up a harmonised framework of thoracic oncology education and its better implementation throughout Europe, both in countries with higher and lower financial capacities

To adequately meet the varying interests and expectations of the aforementioned targeted individuals, we will perform regular need assessments via surveys and other social media means, from which we will derive target groupspecific educational formats. Feedback loops will be used to improve the future quality of our CPD programme, including the involved faculty. Likewise, we will constantly track and appraise new scientific and political developments in the field of thoracic oncology in order to provide up-to-date and evidence-based professional development.

\section{Contextual basis and educational formats}

We will use our thoracic oncology CPD syllabus as the contextual basis of our educational programme. This recently developed catalogue stretches from carcinogenesis, through symptoms, imaging, multidisciplinary team care, systemic therapy and malignant pleural effusion, covering the thoracic oncology trajectory in 32 modules as shown in table 1. Each module has been created via a fixed route: design, creation, internal revision, adaptation, external revision and final adaptation. Each module will be maintained and regularly updated according to future developments by a faculty composed of early career members and senior scientific specialists in thoracic oncology.

The platform used to provide these thoracic oncology educational modules is a comprehensive and adaptive international learning hub with the application of new digital techniques provided by the ERS. We will apply new and well-established ERS educational formats, particularly the following.

- Electronic learning: continuing medical education (CME) online modules and CME tests

- ERS courses: skills and online

- Online and printed publications: ERJ Open Research, the European Respiratory Review, Breathe and the ERS Monograph, with a 
Table 1 Module-based CPD training

\begin{tabular}{|c|c|}
\hline Module 1 & Carcinogenesis, immunology and defence mechanisms \\
\hline Module 2 & Tobacco: risk factors and epidemiology \\
\hline Module 3 & Indoor and outdoor pollution \\
\hline Module 4 & Respiratory hazards associated with occupational factors \\
\hline Module 5 & Asbestos-related diseases \\
\hline Module 6 & Lung cancer screening \\
\hline Module 7 & Signs and symptoms \\
\hline Module 8 & Imaging techniques \\
\hline Module 9 & Bronchoscopy \\
\hline Module 10 & Advanced endoscopy \\
\hline Module 11 & Thoracoscopy and pleural diagnostics \\
\hline Module 12 & Evaluation of patient fitness for diagnostics and therapy \\
\hline Module 13 & Pathology \\
\hline Module 14 & Multidisciplinary team and multidisciplinary team meeting \\
\hline Module 15 & Thoracic surgery \\
\hline Module 16 & Radiotherapy \\
\hline Module 17 & Systemic pharmacotherapy \\
\hline Module 18 & Immunotherapy \\
\hline Module 19 & Rehabilitation \\
\hline Module 20 & Smoking prevention and cessation \\
\hline Module 21 & Palliative care including treatment of tumour-related symptoms and complications \\
\hline Module 22 & Patient and family support \\
\hline Module 23 & Management of paraneoplastic syndromes \\
\hline Module 24 & Thromboembolic disease in thoracic oncology \\
\hline Module 25 & Thoracocentesis including chest tube and tunnelled indwelling pleural catheter \\
\hline Module 26 & Common side effects of systemic therapies and their management \\
\hline Module 27 & Common radiation-induced side effects and their management \\
\hline Module 28 & Solitary pulmonary nodules \\
\hline Module 29 & Malignant pleural mesothelioma \\
\hline Module 30 & Mediastinal tumours \\
\hline Module 31 & Common metastatic pulmonary tumours \\
\hline Module 32 & Malignant pleural effusion \\
\hline
\end{tabular}

longer-term aim of introducing an ERS Handbook of Thoracic Oncology

- Events: ERS International Congress, satellite meetings, research seminars, ERS Live and Best of ERS

- Online blogs: ERS Respiratory Digest

- Social media: quizzes

- HERMES examinations

We will involve prestigious senior faculty and Europe's top-tier thoracic oncology services, but also provide committed early career members with sufficient opportunities to participate in our educational formats, since the latter represent our future.
A general description of the named ERS educational formats can be found on the ERS website: https://www.ersnet.org/professionaldevelopment/cpd/cpd-thoracic-oncology. An outline of our planned thoracic oncology-specific educational formats in 2019/2020 is depicted in table 2. The educational platform of our Thoracic Oncology Assembly, with regular updates, can be viewed on the ERS website as well.

\section{Education recognition}

We aim to gain education recognition in multiple ways. First, high quality of each module should be pursued; then, we aim to obtain international, 


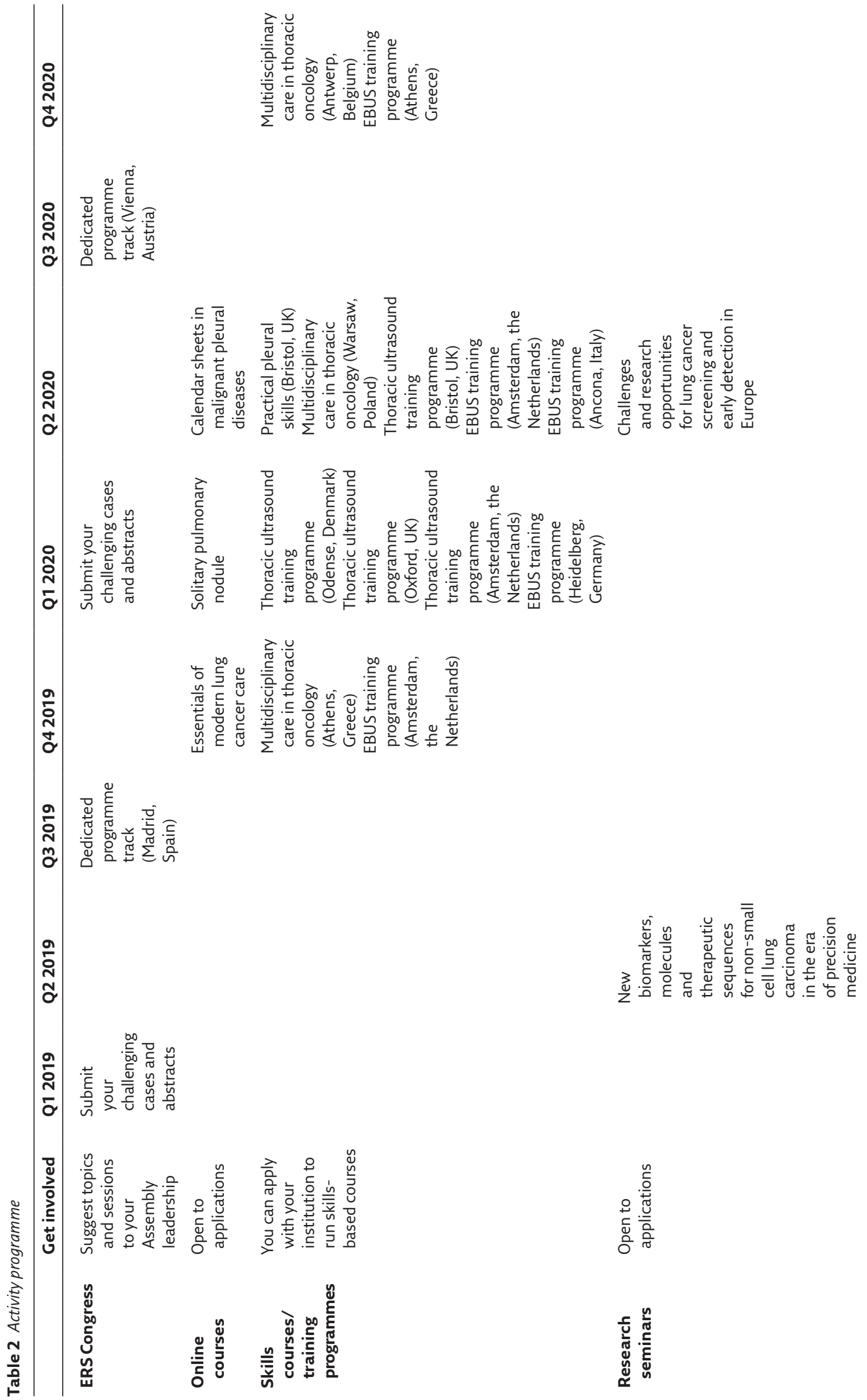




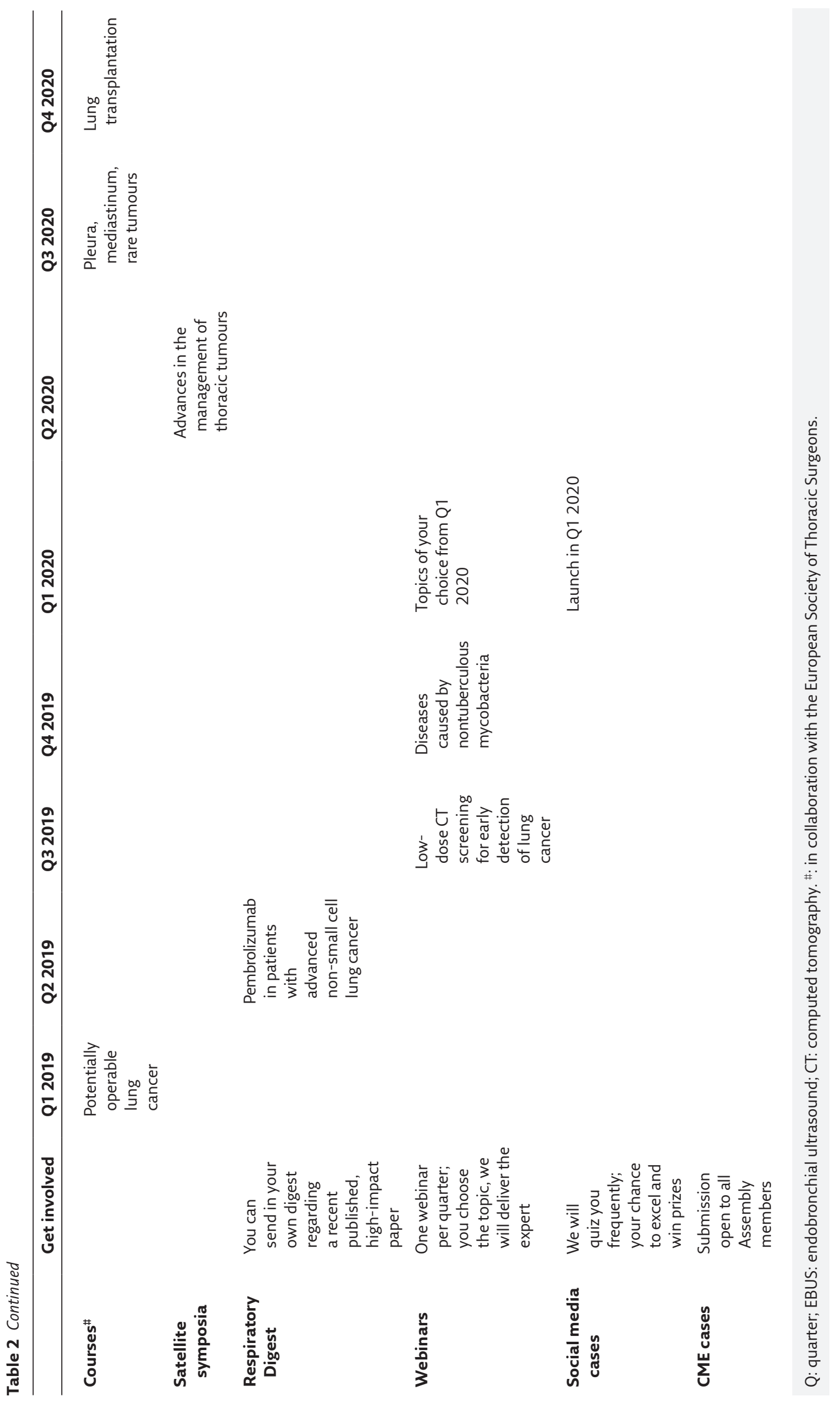


standardised quality certifications, such as CME. Such recognition is an important step in the implementation strategy and will strengthen the communication strategy, both of which are essential to reach out to as much as possible of the target audience. Similar to the successfully established sleep medicine programme [16], we strive to facilitate implementation of our educational initiative into existing national training programmes for thoracic oncology. This will allow recognition of elevating international standards in this important and rapidly changing field of respiratory medicine. It will also allow the certificate of completion of this CPD programme to be a quality-measuring instrument when employing respiratory physicians or researchers from abroad, and thus promote the transfer of knowledge inside Europe and beyond.

\section{Communication}

In an age of transition from analogue towards digital tools, a digital platform is essential to communicate with the target audience and the participants. The ERS already has an excellent basis for this activity to enhance participation and recognition of the thoracic oncology CPD programme. We envision stretching out from the ERS base to our international, national and local partners, seeking fruitful and cordial collaboration as well as avoidance of needless duplication of work. Together, we can use all digital and social media to deliver the message and the education itself. Using the available ERS communication channels, we can already be strong, but through partnership, we will be even stronger and more far reaching.

\section{Affiliations}

Wouter H. van Geffen ${ }^{1}$, Torsten G. Blum², Stefano Aliberti' ${ }^{3,4}$, Kevin G. Blyth ${ }^{5}$, Clementine Bostantzoglou $^{6}$, Amy Farr ${ }^{7}$, Bogdan Grigoriu ${ }^{8}$, Georgia Hardavella ${ }^{9}$, Rudolf M. Huber ${ }^{10}$, Nick Maskell ${ }^{11}$, Gilbert Massard ${ }^{12}$, Najib M. Rahman ${ }^{13}$, Daiana Stolz ${ }^{14}$, Jan van Meerbeeck ${ }^{15}$

${ }^{1}$ Dept of Pulmonary Medicine, Medical Centre Leeuwarden, Leeuwarden, The Netherlands. ${ }^{2}$ Lungenklinik Heckeshorn, Helios Klinikum Emil von Behring, Berlin, Germany. ${ }^{3}$ Dept of Pathophysiology and Transplantation, University of Milan, Milan, Italy. ${ }^{4}$ Respiratory Dept, Fondazione IRCCS Ca' Granda Ospedale Maggiore Policlinico, Milan, Italy. ${ }^{5}$ Glasgow Pleural Disease Unit, Queen Elizabeth University Hospital Glasgow, Glasgow, UK. ${ }^{67}$ th Respiratory Medicine Dept and Asthma Center, Athens Chest Hospital "Sotiria", Athens, Greece. 7European Respiratory Society, Lausanne, Switzerland. "Service de médecine interne, Institut Jules Bordet, Brussels, Belgium. ${ }^{9} 10$ th Dept of Respiratory Medicine, Athens Chest Diseases Hospital "Sotiria", Athens, Greece.

${ }^{10}$ Thoracic Oncology Centre Munich, University of Munich, Munich, Germany, member of the German Center for Respiratory Research (DZL-CPC-M). ${ }^{11}$ Academic Respiratory Unit, University of Bristol, Bristol, UK. ${ }^{12}$ Service de Chirurgie Thoracique, Hôpitaux Universitaires de Strasbourg, Strasbourg, France. ${ }^{13}$ Oxford Centre for Respiratory Medicine, University College, Oxford, UK. ${ }^{14} \mathrm{Clinic}$ for Respiratory Medicine and Pulmonary Cell Research, University Hospital Basel, Basel, Switzerland. ${ }^{15}$ Dept of Pulmonology, Antwerp University Hospital, Edegem, Belgium.

\section{Conflict of interest}

W.H. van Geffen reports a grant from Novartis (an institutional grant for an investigator-initiated trial), outside the submitted work. T.G. Blum has nothing to disclose. S. Aliberti reports grants and personal fees from Bayer Healthcare, Aradigm Corporation, Grifols, INSMED and Chiesi, personal fees from Astra Zeneca, Basilea, Zambon, Novartis, Raptor, Actavis UK Ltd and Horizon, outside the submitted work. K.G. Blyth reports a research grant for a pre-edit and edit trial in malignant effusion, plus consultancy work, from Rocket Medical UK Ltd. C. Bostantzoglou has nothing to disclose. A. Farr is an employee of the European Respiratory Society. B. Grigoriu has nothing to disclose. G. Hardavella has nothing to disclose. R.M. Huber reports personal fees from AstraZeneca Germany, Boehringer Ingelheim Germany, BMS Germany, Lilly, Pfizer Germany, Roche Germany, MSD Germany, Takeda, and Celgene, outside the submitted work. N. Maskell. G. Massard has nothing to disclose. N.M. Rahman reports grants and personal fees from Rocket Medical UK, and personal fees from BD Medical and Lung Therapeutics USA, outside the submitted work. D. Stolz has nothing to disclose. J. van Meerbeeck has nothing to disclose.

\section{References}

1. International Agency for Research on Cancer. Cancer today: data visualization tools for exploring the global cancer burden in 2018. http://gco.iarc.fr/today/home. Date last accessed: 23 April 2019.

2. Alt-Epping B, Staritz AE, Simon ST, et al. What is special about patients with lung cancer and pulmonary metastases in palliative care? Results from a nationwide survey. J Palliat Med 2012; 15: 971-977.

3. Ellis J. The impact of lung cancer on patients and carers. Chron Respir Dis 2012; 9: 39-47.
4. Luengo-Fernandez R, Leal J, Gray A, et al. Economic burden of cancer across the European Union: a population-based cost analysis. Lancet Oncol 2013; 14: 1165-1174.

5. Barta JA, Powell CA, Wisnivesky JP. Global epidemiology of lung cancer. Ann Glob Health 2019; 85: 8.

6. Sun S, Schiller JH, Gazdar AF. Lung cancer in never smokers - a different disease. Nat Rev Cancer 2007; 7: 778-790.

7. Siegel RL, Miller KD, Jemal A. Cancer statistics, 2018. CA Cancer I Clin 2018; 68: 7-30. 
8. Tanvetyanon T. Understanding the uncommon thoracic tumors. Cancer Control 2006; 13: 253.

9. Sculier JP, Vansteenkiste J, Schonfeld N, et al. Thoracic oncology in Europe: the ERS action plan by the Thoracic Oncology Assembly. Eur Respir J 2010; 36: 1227-1228.

10. Gaga M, Powell CA, Schraufnagel DE, et al. An official American Thoracic Society/European Respiratory Society statement: the role of the pulmonologist in the diagnosis and management of lung cancer. Am J Respir Crit Care Med 2013; 188: 503-507.

11. Gamarra F, Boffetta P, De Ruysscher D, et al. Thoracic Oncology HERMES syllabus: setting the basis for thoracic oncology training in Europe. Eur RespirJ 2013; 42: 568-571.

12. Meert AP, Noel JL, Gamarra F, et al. The thoracic oncology specialist: curriculum recommendations in thoracic oncology training. Eur Respir J 2016; 48: 628-631.

13. Gamarra F, Noel JL, Brunelli A, et al. Thoracic oncology HERMES: European curriculum recommendations for training in thoracic oncology. Breathe 2016; 12: 249-255.

14. Blum TG, Rich A, Baldwin D, et al. The European initiative for quality management in lung cancer care. Eur Respir J 2014; 43: 1254-1277.

15. Saarenheimo J, Eigeliene N, Andersen $\mathrm{H}$, et al. The value of liquid biopsies for guiding therapy decisions in nonsmall cell lung cancer. Front Oncol 2019; 9: 129.

16. Mitchell S, Riha RL, Rohde G, et al. Continuing professional development: introducing the ERS International Certificate in Respiratory Sleep Medicine. Breathe 2017; 13: 11-14. 